\title{
Omalizumab usage in chronic urticaria and atopic dermatitis: data from South-East province of Turkey
}

\author{
Hülya Nazik, Mehmet Kamil Mülayim, Perihan Öztürk \\ Department of Dermatology, Kahramanmaraş Sütçü İmam University, Kahramanmaraş, Turkey \\ Adv Dermatol Allergol 2019; XXXVI (6): 734-738 \\ DOI: https://doi.org/10.5114/ada.2019.91424
}

\begin{abstract}
Introduction: Omalizumab is a current treatment option for IgE-associated dermatoses, such as urticaria and atopic dermatitis.

Aim: To evaluate the clinical and laboratory findings of patients with chronic urticaria and atopic dermatitis who received omalizumab.

Material and methods: This study was planned as a cross-sectional and retrospective one. One hundred forty-six cases were included in the study. Omalizumab at a dose of $300 \mathrm{mg}$ for patients with chronic urticaria and $600 \mathrm{mg}$ for patients with atopic dermatitis, was given every 4 weeks. The response to the given treatment and remission status of patients were investigated.

Results: Of 146 patients included in the study, 70.5\% $(n=103)$ were female and their mean age was $42.3 \pm 13.8$ (min.-max.: 14-85). Of the patients, 136 had chronic urticaria and 10 had atopic dermatitis. $29.9 \%(n=26)$ of patients with chronic urticaria had a complete response to omalizumab treatment, whereas $40 \%(n=4)$ of patients with atopic dermatitis had remission.

Conclusions: The decline of complaints with different treatment schemes in different patients suggests that treatment should be designed individually and that personal biomarkers playing a role in the effectiveness of the drug are important. Patients with the high IgE and eosinophil percentages and positive autologous serum test had a better treatment response to omalizumab treatment. Meanwhile, remission was found to be more frequent in atopic dermatitis patients with omalizumab treatment compared to the patients with urticaria. It may be more appropriate to choose patients based on certain criteria to ensure cost-effectiveness in omalizumab treatment.
\end{abstract}

Key words: omalizumab, chronic urticaria, atopic dermatitis, immunoglobulin E.

\section{Introduction}

IgE is an immunoglobulin that has an important role in acute allergic reactions and chronic inflammatory allergic diseases. Omalizumab is a recombinant, humanized antibody developed against immunogloibulin E (IgE). Omalizumab affects by regulating the high-affinity lgE receptor Fc\&R, and reducing the amount of free lgE, mast cell and basophil activation [1]. It has been approved by the Food and Drug Administration (FDA) for treatment of moderate severe asthma and chronic spontaneous urticaria that does not respond to $\mathrm{H} 1$ antihistamines. Meanwhile, omalizumab was used as an off-label in some IgE-related diseases and successful results have been reported. Moreover, it has been reported that omalizumab improved clinical symptoms in diseases such as systemic mastocytosis, atopic dermatitis, food allergy, allergic rhinitis, nasal polyposis and keratoconjunctivitis [2].

\section{Aim}

The aim of this study is to present clinical and demographic data of patients with chronic urticaria (CU) and atopic dermatitis (AD) treated with omalizumab.

\section{Material and methods}

From June 2014 to November 2017, 146 patients, diagnosed with CU and AD, who received omalizumab treatment, were evaluated. The patients' files were scanned and demographic, laboratory and clinical data were obtained retrospectively. The approval for the study

Address for correspondence: Assist. Prof. Hülya Nazik, Department of Dermatology, Kahramanmaraş Sütçü Imam University, 46100 Kahramanmaraş, Turkey, e-mail: dr.hulyagul@hotmail.com Received: 18.05.2018, accepted: 13.08.2018. 
was obtained from the Ethics Committee of the Medical Faculty of Kahramanmaraş Sütçü Imam University (the decision date: 20.12.17, decision no. 6, session: 2017/21). The study was conducted in accordance with the Declaration of Helsinki. Urticaria, which lasted longer than 6 weeks, with or without angioedema, was considered as CU. The diagnosis of AD was made according to anamnesis, physical examination and laboratory findings. The pre-treatment IgE level, thyroid-stimulated hormone (TSH) value and percentage of eosinophils were recorded. The duration of disease, the presence of angioedema, previous and concurrent treatments were investigated. The number of total omalizumab injections administered to the patient, the number of injections after which the treatment was effective, the number of injections after which recurrence was seen and the duration of treatment discontinuation were determined. The clinical response of omalizumab was assessed as follows: those who continued to receive antihistamines in a licensed dose after omalizumab discontinuation were recorded as "complete response", patients who were able to control their complaints with omalizumab together with antihistamines were recorded as "partial response" and patients whose complaints continued despite omalizumab treatment with antihistamines were recorded as "no response". The absence of any antihistamine treatment for at least 6 months after omalizumab treatment was considered as "remission" [3]. Patients with AD who had a normal skin appearance for at least 6 months and who did not need antihistamines were considered as "remission" [4]. The last status was evaluated as the end of treatment, ongoing treatment, and the follow-up discontinuation. The applied doses and complications of omalizumab were recorded. Omalizumab was given once a month by subcutaneous administration of $300 \mathrm{mg}$ for patients with CU and $600 \mathrm{mg}$ for patients with AD. $300 \mathrm{mg}$ of omalizumab was given every 3 weeks to 8 patients with complaints, which were increased during the last week of the 4-week interval. Patients whose treatment had been terminated due to a complete response were followed up until July 2018. The time from the complete response to the determination of complaints and the remission prevalence was identified.

Table 1. Demographic and laboratory data of the patients

\begin{tabular}{|c|c|c|}
\hline Parameter & $\begin{array}{c}\text { Chronic urticaria } \\
(n=136) \\
\text { Mean } \pm \text { SD (min.-max.) }\end{array}$ & $\begin{array}{c}\text { Atopic dermatitis } \\
(n=10) \\
\text { Mean } \pm \text { SD (min.-max.) }\end{array}$ \\
\hline Age [year] & $41.9 \pm 13.2(14-85)$ & $47.5 \pm 19.7(18-73)$ \\
\hline $\operatorname{lgE}[\mathrm{IU} / \mathrm{ml}]$ & $230.6 \pm 361.1(17-2180)$ & $1496.4 \pm 2883.6(48-9270)$ \\
\hline \% Eosinophil & $2.1 \pm 2.0(0-12.1)$ & $5.0 \pm 2.2(1.9-8.8)$ \\
\hline
\end{tabular}

\section{Statistical analysis}

SPSS v.17.0 package program was used for statistical evaluation of data obtained in the study (SPSS Inc, Chicago, Illinois, USA). Continuous data were summarized as mean \pm standard deviation, while categorical data were summarized as number and percentage.

Student's $t$ test was used to compare the IgE and eosinophil percentages of patients with a complete and partial response. The $\chi^{2}$ test was used to compare the relationship between the autologous serum skin test and treatment response.

\section{Results}

Of 146 patients receiving omalizumab treatment, $70.5 \%(n=103)$ were female and $29.5 \%(n=43)$ were male. Of cases, $93.2 \%(n=136)$ were patients with CU and $6.8 \%(n=10)$ with $A D$ and the mean age was 42.3 \pm 13.8 years (min.-max.: 14-85). The mean duration of dis$47.1 \%(n=64)$ of patients with CU reported the angioedema attack at least once. In 4 (2.7\%) patients with $\mathrm{CU}$ receiving omalizumab treatment, the value of thyroid stimulating hormone was outside of the reference range $(0.35-5.5 \mathrm{mIU} / \mathrm{l}) .6 .6 \%(n=9)$ of CU cases were treated because of thyroid pathology. Thyroid pathology was not detected in AD cases. Of $31 \mathrm{CU}$ patients who underwent an autologous serum test, 11 (35.5\%) patients had positive results. Of patients with the positive test result, $8(72.7 \%)$ patients had a complete response and $3(27.3 \%)$ patients had a partial response. A complete response was found to be statistically quite significant in patients with the positive autologous serum test $(p=0.001)$. The demographic and laboratory data of the patients are presented in Table 1. The initial percentages of IgE and eosinophils of patients with CU and AD who had the complete and partial response with omalizumab treatment are presented in Table 2.

$25.3 \%(n=37)$ of the cases used at least two different $\mathrm{H} 1$ antihistamines in the licensed dose before omalizumab treatment. The rest of the cases used systemic corticosteroids and $\mathrm{H} 2$ receptor blockers for less than 20 days in addition to $\mathrm{H} 1$ antihistamines. Of the patients who used steroids and antihistamines, 6 used leukotriene antagonist (LTA), 12 used ketotifen, 2 used doxepin and 5 used cyclosporin. All 10 patients with AD (100\%) received oral antihistamine, topical moisturizer and topical steroid treatment, and 5 (50\%) patients received narrowband UVB treatment for 6 months. Omalizumab treatment was initiated when remission was not achieved despite the use of the indicated treatments.

Chronic urticaria cases received omalizumab injections on average $9.5 \pm 7.0$ times (min.-max.: $1-32$ ) and $A D$ cases received omalizumab injections $4 \pm 2.5$ times (min.max.: 1-7). The treatment with omalizumab was effective with an average of $1.2 \pm 0.6$ (min. - max.: $1-4$ ) injections ease was $53 \pm 52.9$ months (min. - max.: 3-240 months). 
Table 2. The initial levels of IgE and eosinophils in patients who had the complete and partial responses

\begin{tabular}{lcccc}
\hline Disease & Biomarker & Complete response & Partial response & $P_{\text {-value* }}$ \\
\hline $\begin{array}{l}\text { Chronic urticaria } \\
(n=136)\end{array}$ & $\operatorname{lgE}[\mathrm{IU} / \mathrm{ml}]$ & $250.6 \pm 417.4$ & $180.9 \pm 225.6$ & 0.294 \\
\cline { 2 - 5 } & $\%$ Eosinophil & $2.3 \pm 2.1$ & $1.8 \pm 1.7$ & 0.086 \\
\hline $\begin{array}{l}\text { Atopic dermatitis } \\
(n=10)\end{array}$ & IgE $[\mathrm{IU} / \mathrm{ml}]$ & $1939.7 \pm 4098$ & $1053.1 \pm 1193.8$ & 0.663 \\
\hline
\end{tabular}

*Student $t$ test was used.

in urticaria cases and an average of $1.1 \pm 0.6$ (min.-max.: $0-2)$ injections in AD cases. 87.7\% $(n=128)$ of patients receiving omalizumab did not have recurrence during the treatment. In the remaining cases, complaints increased after an average of $7.8 \pm 2.3$ (min.-max.: 3-24) injections. Of the cases, $88.4 \%(n=129)$ were treated without interruption, while $11.6 \%(n=17)$ cases had on average 2.4 \pm 0.5 (min.-max.: 1-3) months' intervals. The treatment response of the patients was evaluated. Sixty-four percent $(n=87)$ of the patients had a complete response, $33.8 \%(n=46)$ had a partial response, and 2.2\% $(n=3)$ had no response. $50 \%(n=5)$ of AD cases had a complete response and another $50 \%(n=5)$ had a partial response. When the drug-related complications were evaluated, it was noticed that tachycardia developed in a single case. Meanwhile, when the final status of patients receiving omalizumab treatment was evaluated, it was learnt that $59.6 \%(n=81)$ of the patients with CU terminated the treatment, $30.9 \%(n=42)$ continued the treatment and $9.6 \%(n=13)$ discontinued the follow-up. When it comes to AD cases, the treatment was terminated because a complete response was obtained in half of the AD cases. While $30 \%(n=3)$ of the patients continued the treatment, $20 \%(n=2)$ discontinued the follow-up. The mean time of complaints in patients with $\mathrm{CU}$ whose treatment was terminated due to a complete response was $10.02 \pm 5.03$ months (min. - max.: 6-42 months). The remission was detected in $29.9 \%(n=26)$ of CU patients with a complete response and in $40 \%(n=4)$ of patients with AD.

\section{Discussion}

Omalizumab is a monoclonal lgG1 antibody that specifically binds to the FceRI domain of serum IgE. Omalizumab inhibits IgE binding to the high-affinity receptors on mast cells, basophils, macrophages and dendritic cells. At the same time, the serum reduces free IgE levels and reduces the IgE receptor level [5]. The use of omalizumab has been approved for chronic spontaneous urticaria not responding to $\mathrm{H} 1$ antihistamines for ages 12 and above [6]. Chronic spontaneous urticaria is characterized by itchy, red, and puffy lesions that appear as attacks for 6 weeks or longer without any reason. According to the international urticaria guidelines, the first choice in the treatment of chronic spontaneous urticaria should be the second generation of $\mathrm{H} 1$ antihistamines at the licensed dose. In cases where treatment is not responsive, in the second-line treatment, the dose of the drug is 4 times higher. If the dose increase is not effective, one of antagonists (omalizumab, cyclosporine and leukotriene) is recommended for the third-line treatment [7].

According to the literature about the usage of omalizumab in CU cases, it has been reported that $150 \mathrm{mg}$ of omalizumab was an effective dose in some patients, while some studies have suggested that $300 \mathrm{mg}$ is the optimal dose [8, 9]. In our clinic, 300 mg of omalizumab was used monthly in patients with CU. However, in 8 cases, $300 \mathrm{mg}$ was used every 3 weeks due to the increase in complaints during the fourth week. The decline of complaints with different treatment schemes in different patients suggests that treatment should be designed individually and that personal biomarkers playing a role in the effectiveness of the drug are important.

It is known that CU is seen more frequently in women and more frequently in third and fourth decades. In this study, in accordance with the literature, $71.3 \%$ of CU patients were female and their mean age was 41.9. In a study conducted by Kim et al., urticaria-associated comorbidities such as allergic rhinitis, drug allergy, asthma, thyroid disease, and cancer were found [10]. In this study, $6.1 \%(n=11)$ of the cases had thyroid pathology.

The relationship between IgE and eosinophil percentage and diseases was investigated. A study reported that the percentage of eosinophils was significantly higher in patients with $A D$ and correlated with clinical severity of the disease [11]. In another study, serum IgE levels were found to correlate with the prevalence of lesions [12]. In a study investigating the aetiology of urticaria, it was found that there was no significant difference in IgE and eosinophil percentages between different urticarial forms [13]. The results of our study were consistent with the literature. The effect of IgE and eosinophil percentage on the response to omalizumab treatment was evaluated. It was seen that these biomarkers were higher in patients with a complete response. In this study, it was concluded that patients with CU and AD with a high percentage of IgE and eosinophil respond better to treatment with omalizumab.

The positivity of the autologous serum test supports autoimmune urticaria. In one study, it was suggested that the use of omalizumab would be beneficial in pri- 
mary treatment-resistant cases rather than immunosuppressive treatment in the treatment of autoimmune urticaria [14]. In this study, consistent with the literature, cases with a positive autologous serum test responded better to omalizumab treatment.

In a study conducted, omalizumab was reported to be effective after 3 days of treatment and continued its effect for 4 weeks [15]. Consistent with the literature, in this study, itching and rash complaints decreased after on average 1.2 injections in patients with CU and 1.1 injections in patients with $A D$. It is recommended that the rate and pattern of healing during 1-3 and 3-6 months of treatment with omalizumab be evaluated. If there is no treatment response in the first 3 months, the diagnosis should be reconsidered and the treatment should be continued if there is a partial response [6].

It has been reported in one study that omalizumab maintains its efficacy up to 3 years after the treatment [16]. In this study, 26 (29.9\%) patients were found to have a remission in the follow-up of 6 months. LarenasLinnemann et al. reported that remission was observed in about $20 \%$ of chronic spontaneous urticarial cases without recurrence during the 1-year follow-up [16]. The high rate of remission in our study may be related to the short follow-up period. The remission was observed in $4(40 \%)$ of 10 AD patients with a complete response. As a result, it can be said that patients with $A D$ with higher percentages of IgE and eosinophil respond better to omalizumab treatment than patients with urticaria.

Immunoglobulin E plays an important role in the pathogenesis of many allergic diseases. Omalizumab has been shown to be effective in the treatment of allergic rhinitis, $A D$, physical urticaria, mast cell dysfunction, food allergies and other allergic diseases [17]. The use of omalizumab in $\mathrm{AD}$ was presented as case reports in the literature. In this study, 10 cases with AD treated with omalizumab treatment were evaluated. Therefore, we think that the use of omalizumab in $A D$ will contribute to the literature. It has been reported that omalizumab reduces the severity of the disease and improve the quality of life in the treatment of AD, especially when it is used in conjunction with classical treatments [5]. In this study, a complete response was achieved in $50 \%$ of cases and remission was achieved in $40 \%$.

It is known that omalizumab has been well tolerated since 2005 in atopic asthma and since 2014 in CU treatment. Side effects such as headache, temporary hair loss and increased blood sugar have been reported in the case reports [18-20]. In this study, only one case developed tachycardia after the injection.

\section{Conclusions}

Omalizumab has become a preferred treatment for chronic and itchy diseases such as urticaria and AD with the effective treatment results and the low side effect profile. The limitation of our studies is that patients are not evaluated with a scoring such as urticaria activity score and AD severity index and that information was obtained from the file records. Patients with high IgE and eosinophil percentages and a positive autologous serum skin test had a better treatment response to omalizumab. Meanwhile, remission is found to be more frequent in $A D$ patients with omalizumab treatment compared to the patients with urticaria. It may be more appropriate to choose patients based on certain criteria to ensure cost-effectiveness in omalizumab treatment.

\section{Conflict of interest}

The authors declare no conflict of interest.

\section{References}

1. Metz M, Staubach P, Bauer A, et al. Clinical efficacy of omalizumab in chronic spontaneous urticaria is associated with a reduction of FcepsilonRI-positive cells in the skin. Theranostics 2017; 7: 1266-76.

2. Navinés-Ferrer A, Serrano-Candelas E, Molina-Molina GJ, Martín M. IgE-related chronic diseases and anti-lgE based treatments. J Immunol Res 2016; 2016: 8163803.

3. Neema S, Chatterjee M. Omalizumab for management of refractory urticaria: experience of a tertiary care centre in Eastern India. Indian J Dermatol 2018; 63: 66-9.

4. Abuabara K, Margolis DJ, Langan SM. The long-term course of atopic dermatitis. Dermatol Clin 2017; 35: 291-7.

5. Switlyk SA, Taylor AL, Gaertner EM. Severe refractory atopic dermatitis with elevated serum IgE treated with omalizumab. Cutis 2017; 99: 6-8.

6. Ferrer M, Boccon-Gibod I, Gonçalo M, et al. Expert opinion: defining response to omalizumab in patients with chronic spontaneous urticaria. Eur J Dermatol 2017; 27: 455-63.

7. Zuberbier T, Aberer W, Asero RZ, et al. European Academy of Allergy and Clinical Immunology; Global Allergy and Asthma European Network; European Dermatology Forum; World Allergy Organization. The EAACI/GA(2) LEN/EDF/WAO Guideline for the definition, classification, diagnosis, and management ofurticaria: the 2013 revision and update. Allergy 2014; 69: 868-87.

8. Kulthanan K, Tuchinda P, Likitwattananurak C, et al. Does omalizumab modify a course of recalcitrant chronic spontaneous urticaria? A retrospective study in Asian patients. J Dermatol 2017; 28: 160-5.

9. Saini S, Rosen KE, Hsieh HJ, et al. A randomized, placebocontrolled, dose-ranging study of single-dose omalizumab in patients with $\mathrm{H} 1$-antihistamine-refractory chronic idiopathic urticaria. J Allergy Clin Immunol 2011; 128: 567-73.

10. Kim BR, Yang S, Choi JW, et al. Epidemiology and comorbidities of patients with chronic urticaria in Korea: a nationwide population-based study. J Dermatol 2018; 45: 10-6.

11. Jenerowicz D, Czarnecka-Operacz M, Silny W. Peripheral blood eosinophilia in atopic dermatitis. Acta Dermatovenerol Alp Pannonica Adriat 2007; 16: 47-52.

12. Katoh N, Hirano S, Kishimoto S. Prognostic factor of adult patients with atopic dermatitis. J Dermatol 2008; 35: 477-83.

13. Machura E, Jońska-Golus M, Krakowczyk H, et al. Etiology and clinical course of urticaria in hospitalized children. Med Wieku Rozwoj 2013; 17: 64-71. 
14. Poddighe D, De Amici M, Marseglia GL. Spontaneous (autoimmune) chronic urticaria in children: current evidences, diagnostic pitfalls and therapeutic management. Recent Pat Inflamm Allergy Drug Discov 2016; 10: 34-9.

15. Ertaş R, Özyurt K, Yıldız S, et al. Adverse reaction to omalizumab in patients with chronic urticaria: flare up or ineffectiveness? Iran J Allergy Asthma Immunol 2016; 15: 82-6.

16. Larenas-Linnemann DES, Parisi CAS, Ritchie C, et al. Update on omalizumab for urticaria: what's new in the literature from mechanisms to clinic. Curr Allergy Asthma Rep 2018; 18: 33.

17. Stokes J. Anti-IgE treatment for disorders other than asthma. Front Med 2017; 4: 152

18. Cordeiro Moreira AS, Rosmaninho Lopes de Soares E Silva MI, Pereira Guilherme MA, et al. Use of omalizumab in the treatment of chronic urticaria. Eur Ann Allergy Clin Immunol 2016; 48: 242-6.

19. Konstantinou GN, Chioti AG, Daniilidis M. Self-reported hair loss in patients with chronic spontaneous urticaria treated with omalizumab: an under-reported, transient side effect? Eur Ann Allergy Clin Immunol 2016; 48: 205-7.

20. Yalcin AD, Gorczynski RM, Cilli A, Strauss L. Omalizumab (anti-lgE) therapy increases blood glucose levels in severe persistent allergic asthma patients with diabetes mellitus: 18 month follow-up. Clin Lab 2014; 60: 1561-4. 